\title{
Design and implementation of a portable fNIRS embedded system
}

\author{
Diego Agrò ${ }^{1}$, Riccardo Canicattì ${ }^{1}$, Maurizio Pinto ${ }^{1}$, Giuseppe Morsellino ${ }^{1}$, \\ Alessandro Tomasino ${ }^{1}$, Gabriele Adamo ${ }^{1}$, Luciano Curcio ${ }^{1}$, Antonino Parisi ${ }^{1}$, \\ Salvatore Stivala ${ }^{1}$, Natale Galioto ${ }^{1}$, Alessandro Busacca ${ }^{1}$, Costantino Giaconia ${ }^{1}$ \\ ${ }^{1}$ DEIM, Department of Energy, Information Engineering and Mathematical Models, \\ University of Palermo, Viale delle Scienze, Blg. 9, 90128 Palermo, Italy \\ \{diego.agro, alessandro.tomasino, gabriele.adamo, luciano.curcio, antonino.parisi, \\ salvatore.stivala, natale.galioto, costantino.giaconia, alessandro.busacca\}@unipa.it \\ canicatt.riccardo@yahoo.it, mauripin@hotmail.com,g.morsellino84@gmail.com
}

\begin{abstract}
We report on the design, development and operation of a portable, low cost, battery-operated, multi-channel, functional Near Infrared Spectroscopy embedded system, hosting up to 64 optical sources and 128 Silicon PhotoMultiplier optical detectors. The system is realized as a scalable architecture, whose elementary leaf consists of a probe board provided with 16 SiPMs, 4 couples of bi-color LED, and a temperature sensor, built on a flexible stand. The hardware structure is very versatile because it is possible to handle both the switching time of the LED and the acquisition of the photodetectors, via an ARM based microcontroller.
\end{abstract}

\section{Introduction}

Functional Near InfraRed Spectroscopy (fNIRS) is an imaging technique for human brain monitoring. It is employed both in clinical medicine and in research laboratories in order to measure the oxygenation status of the brain. Whereas light in the fNIRS range $(650-900 \mathrm{~nm})$ is poorly absorbed by organic tissues, water and lipid, the main bio-molecular absorbers are blood chromophores, as the case of oxygenated and deoxygenated haemoglobin $\left(\mathrm{HbO}_{2}\right.$ and $\mathrm{Hb}$, respectively). In particular, both concentration variations of the latter provide important information on brain activity. If more than one wavelength is used, oxy-haemoglobin and deoxy-haemoglobin changes can be recovered by using the modified Beer-Lambert law [1].

Different types of fNIRS devices have been developed and reported in literature [2]: continuous wave $(\mathrm{CW})$, time domain (TD) and frequency domain (FD) spectroscopy systems. Among such methods, CW-NIRS system is practically easier to develop than the other two techniques and, therefore, it allows to remarkably reduce the cost, especially when a high number of sensors should be employed. In $\mathrm{CW}$ systems, light is commonly emitted at a constant power level, but in some cases, it could be modulated at a few kilohertz. Anyway, such systems cannot give absolute concentration values - unlike the FD and TD systems - but could only provide relative changes [3]. 
Besides the above-mentioned advantages of CW-fNIRS with respect to other diagnostic techniques, our system benefits of the adoption of Silicon PhotoMultiplier (SiPM) as optical sensor, which show significant properties in terms of gain (around $\left.6 \times 10^{6}\right)$, low operating voltage $(<30 \mathrm{~V})$, ruggedness, compactness, reduced sensitivity with temperature, voltage fluctuations, and magnetic fields versus conventional photomultiplier tubes. Such a hardware novelty greatly allows to improve the features of a standard fNIRS system, in terms of both collecting data stage and goodness of the measurements, but, above all, in terms of human safety and portability. Indeed, such a class of SiPMs exhibits outstanding performance in terms of SNR, even without the need of any cooling system. Their SNR (around $45 \mathrm{~dB}$ at an incident optical power of $11 \mathrm{pW}$ and $28{ }^{\circ} \mathrm{C}$ ) is only $6-7 \mathrm{~dB}$ below the SNR of the Hamamatsu PMT model R928 at room temperature. Furthermore, cooling the SiPM at a temperature of $3{ }^{\circ} \mathrm{C}$, it reaches the PMT SNR values at room temperature, even if the SiPM is biased in the range of 28.7 - 33.5 V, against a bias value up to $950 \mathrm{~V}$ of the PMT [4].

With respect to Avalanche PhotoDiode, PIN or Single Photon Avalanche photodiodes, a SiPM matrix ensures better gain and SNR [5]-[9]. The use of Silicon Photomultipliers in a fNIRS system could potentially increase the spatial resolution.

\section{Hardware}

The purpose of this work is to implement an embedded CW-fNIRS system capable of monitoring haemodynamic signals during brain activity [10]-[13]. The system has been designed in order to cover the entire skull surface, by employing a high number of optical components. Thus, it hosts up to 64 LED sources and 128 SiPM detectors. Moreover, a multiplexing technique has been exploited in order to realize a compact and portable system, with a total power consumption suitable for a battery-operated equipment.

The designed system is based on a scalable architecture, in which every leaf consists of 8 modular probe boards (henceforth, simply named probes) built on a flexible stand, hosting 4 bi-color LED (wavelengths equals to 735 and $850 \mathrm{~nm}$ ) as light sources, 16 SiPMs as photo-detectors and a temperature sensor. The used SiPM is relied on $\mathrm{n} / \mathrm{p}$ technology developed at STMicroelectronics R\&D (Catania, Italy), showing a breakdown voltage of about $28.0 \mathrm{~V}$ and a $3.0 \times 3.0 \mathrm{~mm}^{2}$ active area $(2500$ microcells, $62 \%$ fill factor, and $60 \mu \mathrm{m}$ cell pitch) enclosed in a $5.1 \times 5.1 \mathrm{~mm}^{2}$ package.

Each probe is connected to the main board through a flexible flat cable connector, where a powerful ARM microcontroller $(\mu \mathrm{C})$, able to handle both the LED switching time and the acquisition of SiPM, is hosted. Furthermore, on the main board all the needed supply voltages both for the analog and the digital circuitry are implemented, starting from a unique battery pack. Finally, both a UART/RS232 and UART/USB interfaces are realized in order to deliver the digital data to a personal computer. 


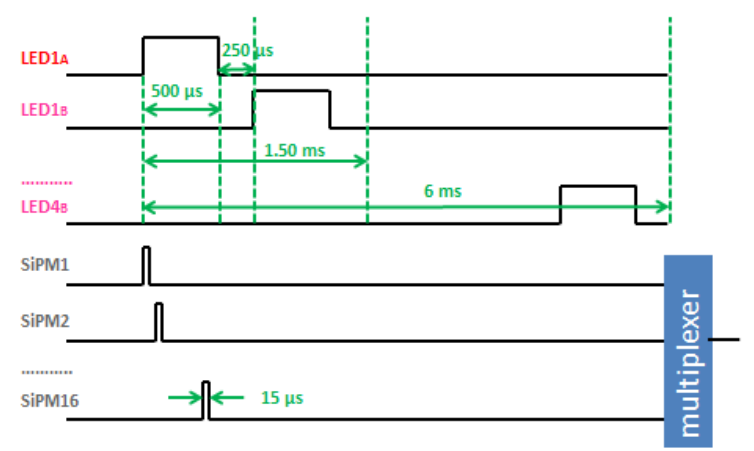

Fig. 1. Timing diagram of the overall fNIRS polling schedule for a single probe sub-platform.

In our CW-fNIRS system, the LED sources illuminate the tissue under test with a constant optical power. Light beam propagates inside the various layers constituting the head, up to the grey matter, where it mostly bends thus the SiPMs are able to capture the scattered and outgoing optical signal. The $\mu \mathrm{C}$ also performs a preliminary data filtering in view of a next post-processing and plotting.

The acquisition process is implemented by following the time sharing polling schedule, presented in Fig. 1. The light sources are enabled one by one and they emit a fixed optical power for $500 \mu \mathrm{s}$, that is the time which the measure process takes place. During this time interval, SiPM selection is sequentially carried out. Each selected SiPM - biased to the working voltage (see later) - gives rise to a photocurrent proportional to the detected optical power. The latter is then acquired through the Analog to Digital Converter (ADC) of the microcontroller, in a time window of $15 \mu \mathrm{s}$. Both SiPM selection and readout are performed by a multiplerdemultiplexer couple, which is handled by the same selection signals coming from the microcontroller. A dead time of $250 \mu \mathrm{s}$, in which all LEDs are off and no readout operations are performed, spaces the lighting of consecutive light sources [14].

However, it is worth highlighting that if more than one probe is connected to the main board, the system is capable to handle several LED-SiPM couples even if such components are positioned in different probes. Such a feature allows a user to optimally handle all LED-SiPM couples, covering the head area under investigation. A relevant property of the probe geometric structure is its repeatability, i.e., the possibility to realize a structure covering the entire head surface of the patient by simply joining all the identical probes. As a result, the chosen geometry is sketched in Fig. 2. 


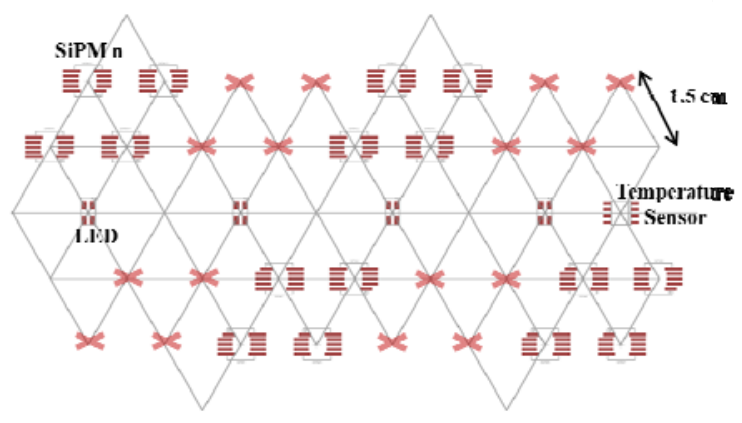

Fig. 2. Probe geometric structure. The red crosses, visible in the picture, represent the positions where sensors of an adjacent probe can be stitched together.

Each of the eight probes is driven by a dedicated circuit, as shown in Fig. 3(a). The latter selects, by means of a de-multiplexer, the correct sequence of LEDs, according to the selection signals coming from the $\mu \mathrm{C}$, as stated by the previous time diagram scheme. The current of the selected LED is controlled via the voltage signal $\left(\mathrm{V}_{\mathrm{DAC}}\right)$ generated by the Digital to Analog Converter (DAC), integrated within the microcontroller, allowing a very fine tuning of the optical power emitted by each LED.

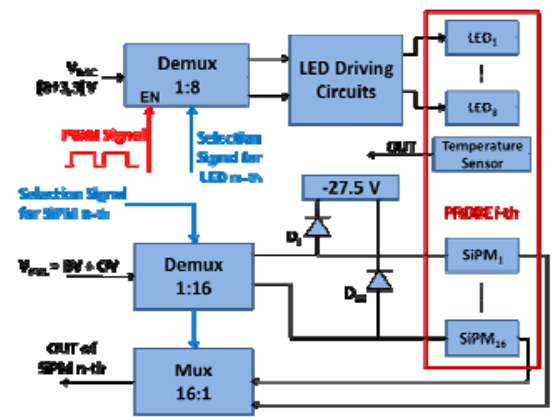

a)

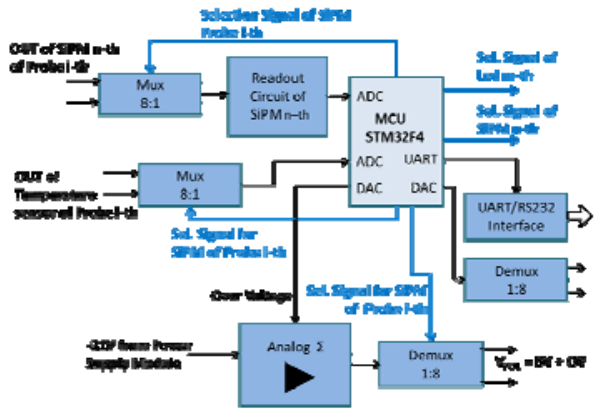

b)

Fig. 3. Block diagram of (a) the circuit handling of i-th probe; (b) the circuit handled by the microcontroller.

As shown in Fig. 3(b), an analog voltage adder generates the SiPM working voltage, by adding a fixed voltage of $-31 \mathrm{~V}$ with a programmable voltage value coming from the DAC module. A dynamic voltage range of $3.3 \mathrm{~V}$ is thus obtained in order to finely control the SiPM Overvoltage $(-27.7 \mathrm{~V} \div-31 \mathrm{~V})$. It is worth noticing that a $-27.5 \mathrm{~V}$ voltage reference is needed in order to keep all the SiPMs at a voltage level just below their threshold, thus obtaining a faster response than the one achieved when $\mathrm{SiPMs}$ are firstly biased at $0 \mathrm{~V}$.

Finally, the whole realized embedded system is shown in Fig. 4. 


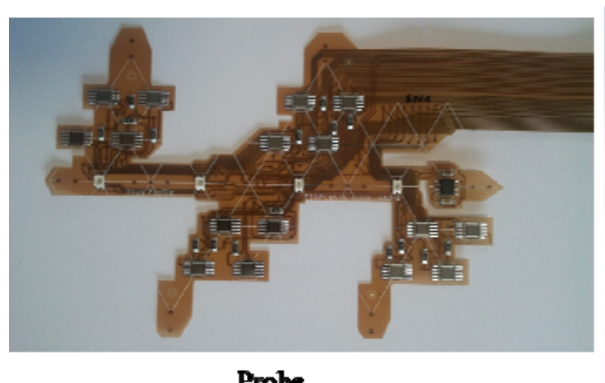

Probe

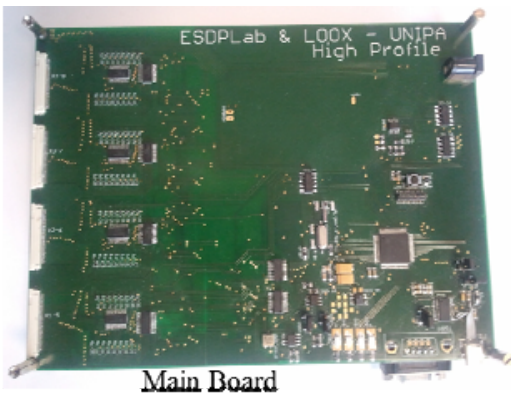

Fig. 4. Picture of the whole fNIRS prototype.

\section{Experimental Results}

A first test has been carried out on a volunteer subject. A probe board was fixed to his forehead by using a black elastic band. Working in these conditions, a set of $200 \mathrm{~s}$ monitoring trials was recorded. During this time window, the subject under test was normally breathing with the exception of the time interval between 70 and $100 \mathrm{~s}$, during which a breath holding phase was set up.

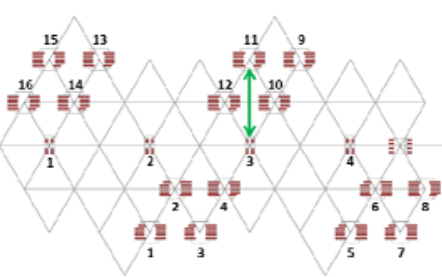

a)

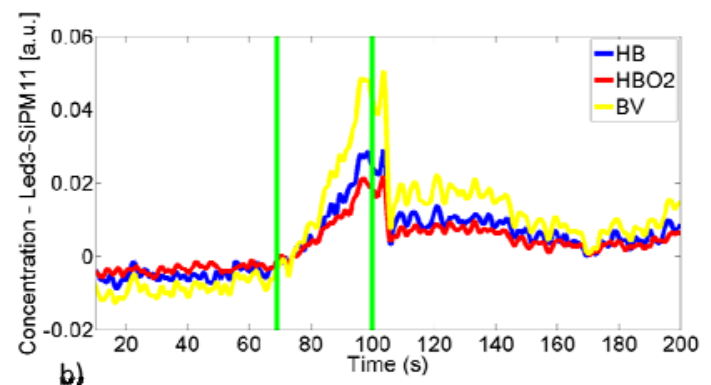

b)

Fig. 5. (a) Probe board pointing out the LED3-SiPM11 couple selected during the experiment; (b) oxygenated (red curves), de-oxygenated (blue curves) and total (yellow curves) haemoglobin levels coming from LED3-SiPM11 couple.

Data shown in Fig. 5(b), coming from the SiPM 11 detector (see Fig. 5(a)), which is positioned at $2.6 \mathrm{~cm}$ from the light source LED 3, have been elaborated by the modified Beer-Lambert law and then they have been filtered by a $300 \mathrm{mHz}$ low-pass filter, in order to cut out the unwanted cardiac pulse (located at about $1.1 \mathrm{~Hz}$ ) and emphasize the oxygenated (red curves, $\mathrm{HBO}_{2}$ ), de-oxygenated (blue curves, $\mathrm{HB}$ ) and total (yellow curves, blood volume, BV) haemoglobin concentration levels. A significant variation of the total haemoglobin is experienced during the breath holding phase. This fact proves that the grey matter of the brain has been reached. In 
particular, this experimental result is in agreement with other similar tests carried out by different researchers and with a completely different equipment [15].

\section{Conclusion}

A CW-fNIRS embedded system prototype has been realized and presented. It hosts up to 64 LED sources and 128 SiPM sensors and it is based on a scalable solution, performing a high level modularity and keeping a low consumption level. Several preliminary functional tests were successfully carried out, thus achieving very encouraging results to be confirmed in the proper validation follow-up. The maximum voltage generated within the prototype is $40 \mathrm{~V}$, so it can be classified as a low voltage device according to the proper regulation $\left(\mathrm{V}_{\max }<50\right.$ Volts @ IEC EN 60601-1). The obtained results by our prototype confirms the high detection performance of the Silicon PhotoMultipliers.

Acknowledgments. This work has been developed in the framework of the ARTEMIS "High Profile" European Funded Project (grant agreement 269356) and it was supported by the Telecom Italia for the Ph.D. program of D. Agrò. We would also like to thank STMicroelectronics of Catania for the SiPMs provision.

\section{References}

1. Bakker, A., Smith, B., Ainslie, P., Smith, K.: Near-Infrared Spectroscopy. Applied Aspects of Ultrasonography in Humans. Prof. Philip Ainslie (Ed.) (2012) 65-89

2. Chance, B., Anday, E., Nioka, S., Zhou, S., Hong, L., Worden, K., Li, C., Murray, T., Ovetsky, Y., Pidikiti, D., Thomas, R.: A novel method for fast imaging of brain function, non-invasively, with light. Optical Express, Vol. 2. (1998) 411-423

3. Rolfe, P.: In Vivo Near Infra-Red Spectrophotometry. Annual Reviews in Biomedical Engineering. Vol. 2 (2000) 315-354

4. Adamo, G., Parisi, A., Stivala, S., Tomasino, A., Agrò, D., Curcio, L., Giaconia, C., Busacca, A., Fallica, G.: "Silicon Photomultipliers Signal-to-Noise Ratio in the Continuous Wave Regime", Selected Topics in Quantum Electronics, IEEE Journal of, Vol. 20, no. 6 (2014)

5. Adamo, G., Agrò, D., Stivala, S., Parisi, A., Giaconia, C., Busacca, A.C., Fallica, G.: SNR measurements of silicon photomultipliers in the continuous wave regime. Photonics West 2014, Silicon Photonics IX (2014) Proc. SPIE 8990, pp. 899016-1-899016-10

6. Adamo, G., Agrò, D., Stivala, S., Parisi, A., Giaconia, C., Busacca, A.C., Mazzillo, M. C., Sanfilippo, D., Fallica, G.: Measurements of Silicon Photomultipliers Responsivity in Continuous Wave Regime. IEEE Transactions on Electron Devices, Vol. 60, n. 11. (2013) $3718-3725$

7. Adamo, G., Agrò, D., Stivala, S., Parisi, A., Curcio, L., Ando', A., Tomasino, A., Giaconia, C., Busacca, A.C., Mazzillo, M. C., Sanfilippo, D., Fallica, G.: Responsivity measurements of $4 \mathrm{H}-\mathrm{SiC}$ Schottky photodiodes for UV light monitoring. Photonics West 2014, Silicon Photonics IX (2014) paper no. 8990-41 
8. Pernice, R., Adamo, G., Stivala, S., Parisi, A., Busacca, A.C., Spigolon, D., Sabatino, M.A., D'Acquisto, L., Dispenza, C.: Opals infiltrated with a stimuli-responsive hydrogel for ethanol vapor sensing. Optical Materials Express, Vol. 3, n. 11. (2013) 1820-1833

9. Adamo, G., Agrò, D., Stivala, S., Parisi, A., Giaconia, C., Busacca, A.C., Mazzillo, M. C., Sanfilippo, D., Fallica, G.: Responsivity measurements of N-on-P and P-on-N silicon photomultipliers in the continuous wave regime. Proc. SPIE 8629, Photonics West 2013, Silicon Photonics VIII, 86291. USA, San Francisco (2013) 86291A-1-86291A-9

10.Sanfilippo, D., Valvo, G., Mazzillo, M., Piana, A., Carbone, B., Renna, L., Fallica, P. G., Agrò, D., Morsellino, G., Pinto, M., Canicattì, R., Galioto, N., Adamo, G., Stivala, S., Parisi, A., Curcio, L., Giaconia, C., Busacca, A. C., Pagano, R., Libertino, S., Lombardo, S.: Design and development of a fNIRS system prototype based on SiPM detectors. Photonics West 2014, Silicon Photonics IX (2014) paper no. 8990-40

11.Xue, H., Bestonzo, M., Acharya, U. R., Molinari, F.: Design and Implementation of a Continuous Wave Near Infrared Spectroscopy System for Bedside and Home Monitoring. Journal of Medical Imaging and Health Informatics, Vol. 1. (2011) 317-324

12.Bozkurt, A., Rosen, A., Rosen, H., Onaral, B.: A portable near infrared spectroscopy system for bedside monitoring of newborn brain. BioMedical Engineering OnLine, Vol. 4. (2005) $1-11$

13.Zimmermann, R., Braun, F., Achtnich, T., Lambercy, O., Gassert, R., Wolf, M.: Silicon photomultipliers for improved detection of low light levels in miniature near-infrared spectroscopy instruments. Biomedical Optics Express, Vol. 4. (2013) 659-666

14.Agrò, D., Adamo, G., Pinto, M., Canicattì, R., Tomasino, A., Parisi, A., Stivala, S., Curcio, L., Giordano, A., Giaconia, C., Busacca, A.: "Design of a portable (CW) fNIRS embedded system", SIMAI 2014 (2014), Taormina, Italy, DOI: 10.13140/2.1.3050.5608

15.Vikrant S.: Near Infrared Spectroscopy: a study of celebral hemodynamics during breathholding and development of a system for hotflash measurement. M.S.Thesis. University of Arlington Texas (2005) 